\title{
Homology Modelling and in silico Characterization of Laccase from Lentinula edodes
}

\author{
Vemula Vani*, Jayashree D J, Navya K S, Akanchha , Syeda Tanzeela Zaman \\ Department of Microbiology, Ramaiah College of Arts, Science and Commerce, Bangalore, Karnataka, INDIA.
}

\begin{abstract}
Introduction: Laccases are phenol oxidases which belong to the superfamily of multicopper oxidases. Laccases are found in almost all wood-rotting fungi. There is evidence that laccases can play an important role in lignin degradation, fruiting body formation, pigment formation during asexual development, competitor interactions and pathogenesis. Laccase from Lentinula edodes is used in variety of applications like to reduce toxicity, partial decolourization of effluent water and decolourization of chemically different dyes like Remazole brilliant blue $R$, bromophenol blue, methyl red and naphtol blue black. The objectives of this study include prediction of three-dimensional (3D) structure of laccase from Lentinula edodes using homology modelling, in silico characterization and analysis of laccase from this organism using computational methods. Methods: The sequence of laccase from Lentinula edodes was retrieved from UniProt database and sequence analysis was carried out using BLAST for the selection of template. The protein 3D structure was modelled using ModWeb server. The obtained 3D model of the laccase from Lentinula edodes was visualized and analyzed using RasMol. The quality of the $3 \mathrm{D}$ structure of protein was verified by its energy and stereochemical properties. The erred regions were remodelled by loop modelling using SWISS PDB viewer. Further, the in sillico characterization of the laccase from Lentinula edodes was computed. Results: The 3KW7 A of Trametes
\end{abstract}

Sp AH28-2 is used as template for model building of laccase from $L$. edodes. The atom model obtained in PDB format showed unstable region in the model. These unstable regions were selected and remodelled by loop modelling. The remodelled structure was further evaluated by its stereochemical quality and energy. The quality of the remodelled structure was found to be improved. Conclusion: Evaluated 3D structure of laccase from $L$. edodes shows that predicted model was of good quality because maximum residues are present in favoured region which indicates that stereochemical quality of predicted 3D structure was reasonably good. It suggests that this model can be used to understand molecular interaction of this laccase with the other proteins.

Key words: Laccase, Lentinula edodes, Homology modelling, In silico, BLAST, MODWEB, Swiss PDB Viewer.

\section{Correspondence}

Dr. Vemula Vani

Assistant Professor, Department of Microbiology, Ramaiah college of Arts, Science and commerce, Bangalore- 54, Karnataka, INDIA.

Phone no: +919632119023

Email: vemula.vani@gmail.com

DOI: 10.5330/ijpi.2020.3.50

\section{INTRODUCTION}

Laccases are copper containing oxidase enzymes found in many plants, fungi and micro-organisms. Laccases were also found in various basidiomycetous and ascomycetous fungi. ${ }^{1}$ The first report of a bacterial laccase was from the Gram-negative soil bacterium Azospirillum lipoferum $^{2}$ and the enzyme was believed to be involved in melanisation. ${ }^{3}$ These enzymes can be used for textile finishing or textile dyeing, teeth whitening and also it has industrial, environmental, diagnostic and synthetic uses. ${ }^{4}$ Laccases can also be used in bioremediation. ${ }^{5}$ Lentinula edodes (shiitake) is one of the world's second largest cultivated medicinal and edible mushrooms used as functional foods. It is used in the treatment of tumors, flu, heart diseases, high blood pressure, obesity, problems related to sexual dysfunction and ageing, diabetes, liver ailments, respiratory diseases, exhaustion and weakness. ${ }^{6} \mathrm{~L}$. edodes is considered to be one of the most valuable medicinal mushrooms. ${ }^{7,8}$ The experimental methods to determine the protein 3D structure like X-ray crystallography, nuclear magnetic resonance spectroscopy are technically demanding, time consuming and may not keep with which new protein sequences are being discovered by genomics research. Although a large number of genes are being discovered, the number of protein structures being solved by experimental methods is limited.

Alternative strategies for structure prediction and modelling of proteins are computational methods. The major computational methods for predicting the structure of proteins are ab initio methods and homology modelling. Homology modelling remains the most accurate prediction method. ${ }^{9}$ It helps to bridge the gap between the available sequences and structural information by providing reliable and accurate protein models. Homology modelling is a technique for predicting or generating detailed $3 \mathrm{D}$ structures of proteins based on coordinates of known homologues.

The main steps to create a Homology model are as follows: 1) Identification of structural homologues. 2) Selection of structural homologues used as templates for modeling. 3) Alignment of templates with the protein sequence to be modelled. 4) Model building. 5) Evaluation and refinement of the model.

The objectives of this present study are to predict the three-dimensional (3D) structure of laccase from Lentinula edodes using homology modelling, in silico characterization and analysis of laccase from this organism using computational methods.

\section{MATERIALS AND METHODS}

\section{Retrieval of laccase sequence of Lentinula edodes from UniProt database}

The sequence details of the laccase from Lentinula edodes was retrieved from UniProt database. The UniProt Knowledgebase (UniProtKB) is the central hub for the collection of functional information on proteins, with accurate, consistent and rich annotation. ${ }^{10}$ 


\section{Identification of Template}

For getting the homologous templates, BLAST ${ }^{11,12}$ was used. In bioinformatics, Basic Local Alignment Search Tool (BLAST) is a sequence similarity search program that can be used via a web interface or as a stand-alone tool. There are several types of BLAST to compare all combinations of nucleotide or protein queries with protein databases. BLAST is a heuristic method that finds short matches between two sequences and attempts to start alignments from these hotspots and also provides statistical information to help decipher the biological significance of the alignment. ${ }^{13}$

\section{Secondary structure prediction}

SOPMA was used for secondary structure prediction of laccase from $L$. edodes. SOPMA (Self - Optimized Prediction Method with Alignment) is an improvement of SOPM method. ${ }^{14}$ SOPMA correctly predicts $69.5 \%$ of amino acids for a three-state description of the secondary structure (alpha-helix, beta-sheet and coil) in a whole database containing 126 chains of non-homologous (less than $25 \%$ identity) proteins. ${ }^{15}$

\section{Model building}

For building the models, ModWeb server was used. ${ }^{16}$ ModWeb is a comparative modelling webserver for protein structure modelling. ModWeb accepts one or many sequences in the FASTA format and calculates their models using ModPipe based on the best available templates from the Protein Data Bank (PDB). ModWeb includes a database of annotated comparative protein structure models, containing models for more than 3.8 million unique protein sequences. ModWeb is developed in the laboratory of Andrej Sali at UCSF. ${ }^{17}$ ModWeb models are also accessible through the Protein Model Portal.

\section{Loop modelling}

Modelling of the errored loops in laccase structure from L. edodes was carried out using Swiss-PDB Viewer ver 4.1. Swiss-PDB Viewer is an application that provides a user-friendly interface that allows the analysis of several proteins at the same time. These regions were selected using control panel and suitable loop was selected from the database which assures the stability of the selected loop including the overall structure. ${ }^{18}$

\section{Visualization of the model}

The modelled 3D structure of laccase from L. edodes was visualised and analysed using Rasmol. ${ }^{19}$ RasMol is a computer program written for molecular graphics visualization and used mainly to depict and explore biological macromolecule structures, such as those found in the Protein Data Bank. It was originally developed by Roger Sayle in the early 1990s.

\section{Evaluation of the model}

The evaluation of the modelled 3D structure for laccase from $L$. edodes was carried out using Verify 3D program. The three-dimensional (3D) profile of a protein structure is a table computed from the atomic coordinates of the structure that can be used to score the compatibility of the 3D structure model with any amino acid sequence. Threedimensional profiles computed from correct protein structures match their own sequences with high scores. An incorrectly modelled segment in an otherwise correct structure can be identified by examining the profile score in a moving-window scan. ${ }^{20,21}$ The stereo-chemical quality of the modelled laccase structure from $L$. edodes was analyzed by Ramachandran plot $^{22}$ using the software RAMPAGE. ${ }^{23}$

\section{Computation of Physical and Chemical Properties of Laccase from $L$. edodes}

The physical and chemical properties of laccase from L. edodes was computed by ProtParam, ${ }^{24}$ a tool which allows the computation of various physical and chemical parameters of a given protein sequence. The computed parameters include the molecular weight, theoretical pI, extinction coefficient, estimated half- life, instability index, aliphatic index and grand average of hydropathicity.

\section{RESULTS}

\section{Sequence analysis}

The sequence of the laccase from L. edodes was retrieved from UniProt $\mathrm{KB}$, which is given in FASTA format (Figure 1). This laccase sequence consists of 524 aa and it has a mass of 55,357 Da.

\section{Identification of template}

The sequence which is obtained from the Uniprot $\mathrm{KB}$ was used as the input in BLAST-P server to find out the suitable template for the model building. It was seen that the Lac b from Trametes sp AH28-2 (3KW7A) had $63 \%$ identity among the homologus sequences resulted from the BLAST-P server. It shows the strongest match with the laccase from $L$. edodes. Resolution of this laccase structure is $3.44 \AA$. Sequence length is 502.

\section{Secondary structure prediction of target protein}

The secondary structure of laccase from $L$. edodes was predicted by the improved self-optimized prediction method (SOPMA). The protein sequence of the laccase was given as input and four conformational states, including helices, sheets, turns and coils were analyzed. The predicted secondary structure of the laccase from L. edodes is shown in Figure 2.

\section{Model building}

The refined sequence-sequence alignment as obtained by BLAST- P (Figure 3) was used to construct 3D model of laccase from Lentinula edodes using MODWEB server.

\section{Model evaluation}

A full atom model in PDB format of the laccase from Lentinula edodes was obtained by ModWeb. The model generated from ModWeb was submitted to Verify 3D program and the graph was obtained (Figure 4). Analysis of the graph revealed that some of the regions in the model were not stable and such regions corresponded to the regions of insertion and deletion. These regions (Table 1) were considered for Loop modelling. The stereochemical quality of the model was evaluated using Ramachandran plot (Figure 5).

While loop modelling, for each loop region (Table 1) anchor residues were carefully selected and the loop database of SPDBV was scanned. Of the loops obtained from the database, one was selected on its stereo chemical compatibility and its side chains interaction with the rest of the structure. Loops selected were added to the model one at a time and all the selected loop regions were remodelled. After remodelling the loop regions, the model was subjected to energy minimization using Swiss PDB Viewer.

$>\operatorname{tr|} \mid$ 1CGD5|E1CGD5_LENED Laccase lcc6 OS=Lentinula edodes

GN=lcc6PE $=3 \mathrm{SV}=1 \mathrm{MNFVTALPLIAQLIGTALAAIGPVTDLHVVNKFIQPDGFNRSYI}$ LAEGVFPGPLISGNKGDNFQINVINELTNDTMLLSTSIHWHGLFQGGTNWADGPAF INQCPIAAGNSFLYNFNVPDQAGTFWYHSHLATQYCDGLRGPLVVYDPQDPYLSL YDVDDDSTVITLSDLYHVPAPLIVGAATSDATLINGLGRYTNGPATAPLAVISVTLG KRYRFRLVSISCEPNFVFSIDGHTFTVIEVDGVSHDPVVADSIQIFASQRYSFVLNAN QIVGNYWIRANPSVGTTGFTGGINSAILRYVGAPVADPVTVSTSINPLLETSLHPLV SPGAPGSATLSGVDVDLRLVLGFNAGSFLVNGVKFVPPTVPVLLQILSGATTAASL LPAGSVYTLPLNSTIQLSFDASAVAAIGGPHPFHLHGHNFDVVRPAGSTTYNYANPI RRDTVSTGAATDNVTIRFTTNNAGPWFLHCHIDWHLEAGFAIVLAEDAPGVASAN PTTDAWNDLCPIYDALTSAELGGGGGPTS

Figure 1: Laccase sequence obtained from UniPort KB in FASTA format of from Lentinula edodes. 
After energy minimization, the model was again checked for its stereochemical quality and Verify $3 \mathrm{D}$ graph. In this model, few residues were appeared in disallowed region of Ramachandran plot. In Verify 3D graph also troughs were found, which indicates that the remodelled loops are energetically not stable.

From this model again the errored loops were selected and remodelled using SPDBV. This was continued till we got the model, which satisfied the criteria of Ramachandran plot, Verify 3D graph.

For the finally obtained laccase from L. edodes model (Figure 6), the Verify $3 \mathrm{D}$ graph is shown in (Figure 7 ). The stereochemical quality of the final model was evaluated using Ramachandran plot (Figure 8).

\section{Analysis of Physical and chemical properties of laccase} from $L$. edodes

The analysis of Physical and chemical properties of the laccase from $L$. edodes was carried out using ProtParam tool. The computed parameters including the molecular weight, theoretical pI, extinction coefficient, estimated half-life, instability index, aliphatic index and grand average of hydropathicity are mentioned below;

Total number of negatively charged residues (Asp + Glu): 39

Total number of positively charged residues (Arg + Lys): 18

Number of amino acids: 524

Molecular weight: 55357.42

Theoretical pI: 4.88

Formula: $\mathrm{C}_{2511} \mathrm{H}_{3833} \mathrm{~N}_{655} \mathrm{O}_{746} \mathrm{~S}_{7}$

Total number of atoms: 7752

MNFVTALPLIAQLIGTALAAIGPVTDLHVVNKFIQPDGFNRSYILAEGVFPGPLISGNKGDNFQINVINE

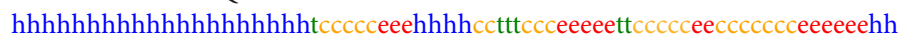
LTNDTMLLSTSIHWHGLFQGGTNWADGPAFINQCPIAAGNSFLYNFNVPDQAGTFWYHSHLATQYCDGLR

ccttceeeecceehtccccccccccccceeeecccccttcceeeeeeccccccceeehhhhhhhttttt GPLVVYDPQDPYLSLYDVDDDSTVITLSDLYHVPAPLIVGAATSDATLINGLGRYTNGPATAPLAVISVT

cceeeecttcceeeeeettttceeeeehheeccccceeeecccchheeehtcccccccccccceeeeee LGKRYRFRLVSISCEPNFVFSIDGHTFTVIEVDGVSHDPVVADSIQIFASQRYSFVLNANQIVGNYWIRA

ttccceeeeeeecctteeeeettteeeeeettccccceehhhhhhhhttheeeechtthhhheeeec NPSVGTTGFTGGINSAILRYVGAPVADPVTVSTSINPLLETSLHPLVSPGAPGSATLSGVDVDLRLVLGF

cttccccccccccchhheeettcccccceeeecccchhhhh cccccccttttcceeetceeeeeeeec NAGSFLVNGVKFVPPTVPVLLQILSGATTAASLLPAGSVYTLPLNSTIQLSFDASAVAAIGGPHPFHLHG

ctteeeetteeecccccceeeehhttcchhhheccttceeeecccceeeeee hhhheeetcccceeecc HNFDVVRPAGSTTYNYANPIRRDTVSTGAATDNVTIRFTTNNAGPWFLHCHIDWHLEAGFAIVLAEDAPG

cceeeecttcceeetccccccceeeccccccceeeeeettccceeeecchhhhhhttheeeeetcctt VASANPTTDAWNDLCPIYDALTSAELGGGGGPTS

Cccccccchhhhhhhhhhhhhhhhhctttcccce

Sequence length: 524

SOPMA:

Alpha helix (Hh): $\quad 96$ is $18.32 \%$

310 helix $(\mathrm{Gg})$ : $\quad 0$ is $0.00 \%$

Pi helix (Ii): $\quad 0$ is $0.00 \%$

Beta bridge $(\mathrm{Bb}): \quad 0$ is $0.00 \%$

Extended strand (Ee): 169 is $32.25 \%$

Beta turn (Tt): $\quad 69$ is $13.17 \%$

Bend region (Ss): $\quad 0$ is $0.00 \%$

Random coil (Cc): 190 is $36.26 \%$

Ambiguous states (?): 0 is $0.00 \%$

Other states: 0 is $0.00 \%$

Figure 2: Secondary structure prediction of laccase from L. edodes.

\begin{tabular}{cccccc}
\hline Score & Expect & Method & Identities & Positives & Gaps \\
\hline $\begin{array}{c}693 \\
\text { bits(1789) }\end{array}$ & 0.0 & $\begin{array}{c}\text { Compositional } \\
\text { matrix adjust. }\end{array}$ & $346 / 504(69 \%)$ & $\begin{array}{c}396 / 504 \\
(78 \%)\end{array}$ & /504(2\%) \\
\hline Query & & & & \multicolumn{2}{c}{20} \\
\hline
\end{tabular}

AIGPVTDLHVVNKFIQPDGFNRSYILAEGVFPGPLISGNKGDNFQINVINELTNDTMLLS 79

AIGPVTDL + N + PDGF R+ ++A GVFPGPLI+GNKGDNFQINVI+ LTN TML + Sbjct 1

AIGPVTDLTISNADVTPDGFTRAAVVANGVFPGPLITGNKGDNFQIN VIDNLTNATMLKT 60

Query

80

TSIHWHGLFQGGTNWADGPAFINQCPIAAGNSFLYNFNVPDQAGTFWYHSHLATQYCDGL 139

T+IHWHGLFQ VPDQAGTFWYHSHL+TQYCDGL

GTNWADGPAF+NQCPIA+GNSFLY+F

Sbjct

61

TTIHWHGLFQHGTNWADGPAFVNQCPIASGNSFLYDFTVPDQAGTFWYHSHLSTQYCDGL 120

Query

140

RGPLVVYDPQDPYLSLYDVDDDSTVITLSDLYHVPAPLIVGAA---TSDATLINGLGRYT 196

RGPLVVYDP DPY S+YDVDDD+TVITLSD YH A L G A +D+ LINGLGR+

Sbjct

121

RGPLVVYDPSDPYASMYDVDDDTTVITLSDWYHTAAKL--GPAFPPNADSVLINGLGRFA 178

Query 197

NGPATAPLAVISVTLGKRYRFRLVSISCEPNFVFSIDGHTFTVIEVDGVSHD-

PVVADSIQ 256

G A+ LAVI+V KRYRFRLVS+SC+PNF FSIDGH T+IEVDGV+H+P+ DSIQ

Sbjct

179

GGNASD-LAVITVEQNKRYRFRLVSLSCDPNFTFSIDGHNMTIIEVDGVNHEPLEVDSIQ 237

Query 257 IFASQRYSFVLNANQIVGNYWIRANPSVGTTGFTGGINSAILRYVGAPVADPV-TVSTSI 315

IFASQRYSFVLNA Q V NYWIRA P+ GT TGG+NSAILRY GA + DP +TS+

Sbjct 238

IFASQRYSFVLNATQSVDNYWIRAIPNTGTIDTTGGLNSAILRYSGADIVDPTANATTSV 297

Query 316

NPLLETSLHPLVSPGAPGSATLSGVDVDLRLVLGFNAGSFLVNGVKFVPPTVPVLLQILS 375

PL+ET L PL SP APG + GVD+ + L FN +F +N +PPTVPVLLQILS

Sbjct

298

IPLVETDLVPLDSPAAPGDPVVGGVDLAMNLDFSFNGTNFFINNETLIPPTVPVLLQILS 357

Query 376

GATTAASLLPAGSVYTLPLNSTIQLSFDASAVAAI----GGPHPFHLHGHNFDVVRPAGS 431

GA + A+ LLP GSVYTLPLNSTI+LSF + V + G PHPFHLHGH F VVR AGS

Sbjct

358

GAQSASDLLPTGSVYTLPLNSTIELSFPITTVNGVTNAPGAPHPFHLHGHAFSVVRSAGS 417

Query 432

TTYNYANPIRRDTVSTGAATDNVTIRFTTNNAGPWFLHCHIDWHLEAGFAIVLAEDAPGV 491

+ YNY NP+RRDTVSTG DNVTIRFTT+NAGPWFLHCHID+HLEAGFAIV AED P

Sbjct

418

SDYNYVNPVRRDTVSTGNPGDNVTIRFTTDNAGPWFLHCHIDFHLEAGFAIVFAEDTPDT 477

Query

492

ASANPTTDAWNDLCPIYDALTSAE 515

AS NP AW+DLCP YDAL ++

Sbjct

478

ASVNPVPTAWSDLCPTYDALDPSD 501

Figure 3: Alignment of template sequence with query sequence (laccase from $L$. edodes) obtained by BLAST. 
Extinction coefficientsExtinction coefficients are in units of $\mathrm{M}^{-1} \mathrm{~cm}^{-1}$, at $280 \mathrm{~nm}$ measured in water.

Ext. coefficient 64080

Abs $0.1 \%$ (=1 g/l) 1.158, assuming all pairs of Cys residues form cystines Ext. coefficient 63830

Abs 0.1\% (=1 g/l) 1.153, assuming all Cys residues are reduced

Estimated half-lifeThe $\mathrm{N}$-terminal of the sequence considered is $\mathrm{M}$ (Met).

The estimated half-life is: $30 \mathrm{hr}$ (mammalian reticulocytes, in vitro). $>20 \mathrm{hr}$ (yeast, in vivo).

$>10 \mathrm{hr}$ (Escherichia coli, in vivo).

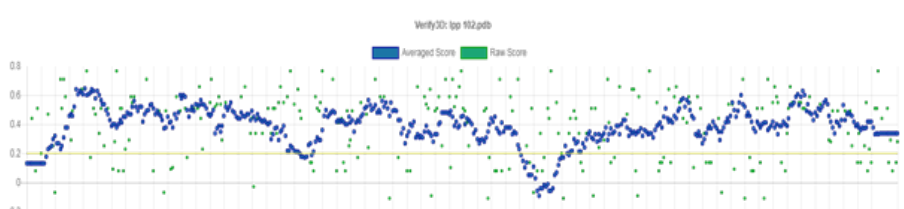

Figure 4: Verify 3D graph of laccase from $L$. edodes before loop modelling.

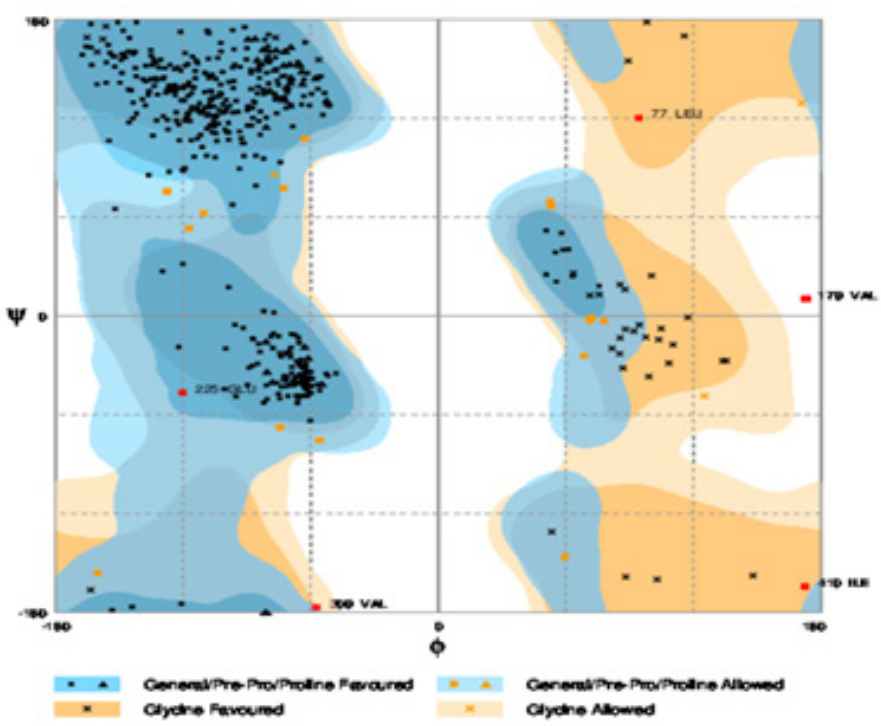

Figure 5: Ramachandran plot of laccase from $L$. edodes before loop modelling.

Number of residues in favoured region ( 98.0\% expected): 469 (94.9\%) Number of residues in allowed region ( $2.0 \%$ expected): 20 (4.0\%) Number of residues in outlier region: $5(1.0 \%)$

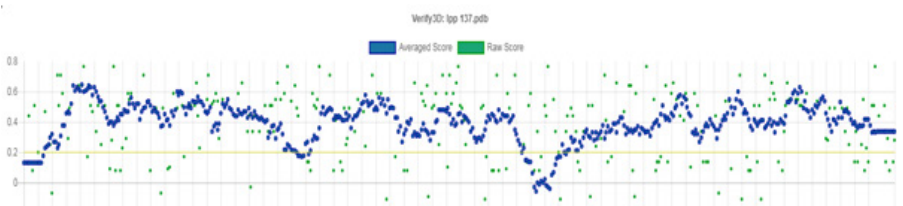

Jols

Figure 7: Verify 3D graph of laccase from L. edodes after loop modelling.
Instability index The instability index (II) is computed to be 25.78 This classifies the protein as stable.

Aliphatic index: 97.71

Grand average of hydropathicity (GRAVY): 0.230

\section{DISCUSSION}

Laccases have the ability to synthesize the products which may be highly valuable from pharmaceutical point of view. In pharmaceutical industry, laccases have two major roles- either they are directly involved in the synthesis of new medicinally valuable compounds by different synthesis methods or applicable for the bioremediation of by- products

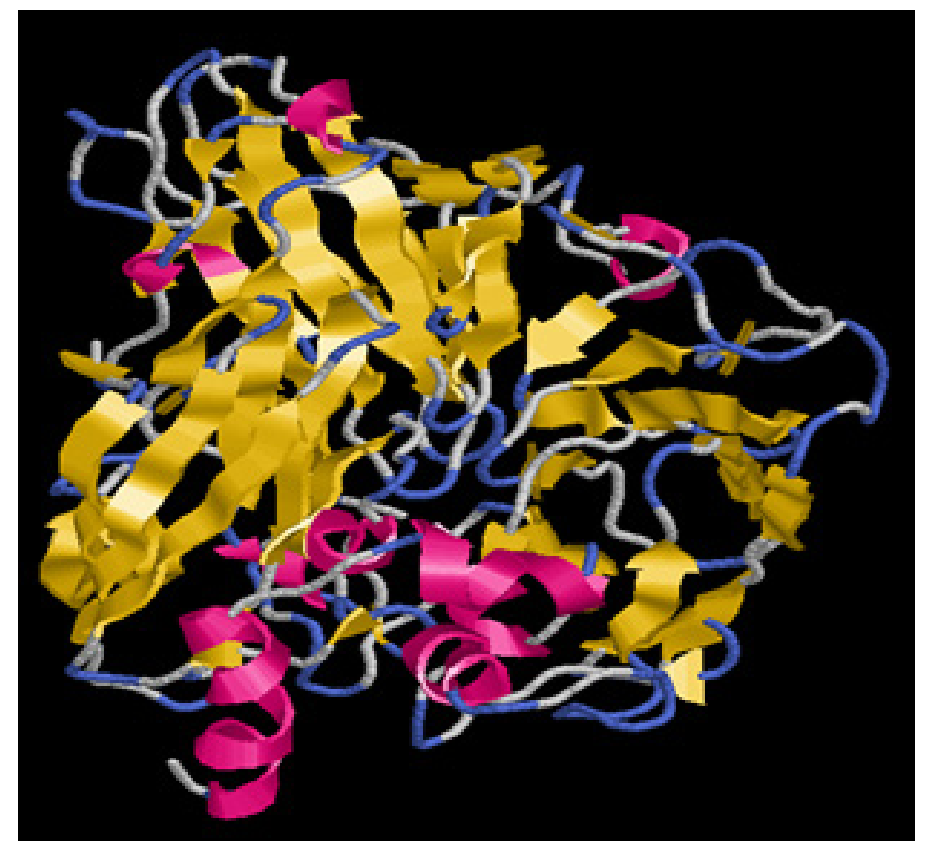

Figure 6: 3D structure of laccase from $L$. edodes after loop modelling as viewed in RasMol.

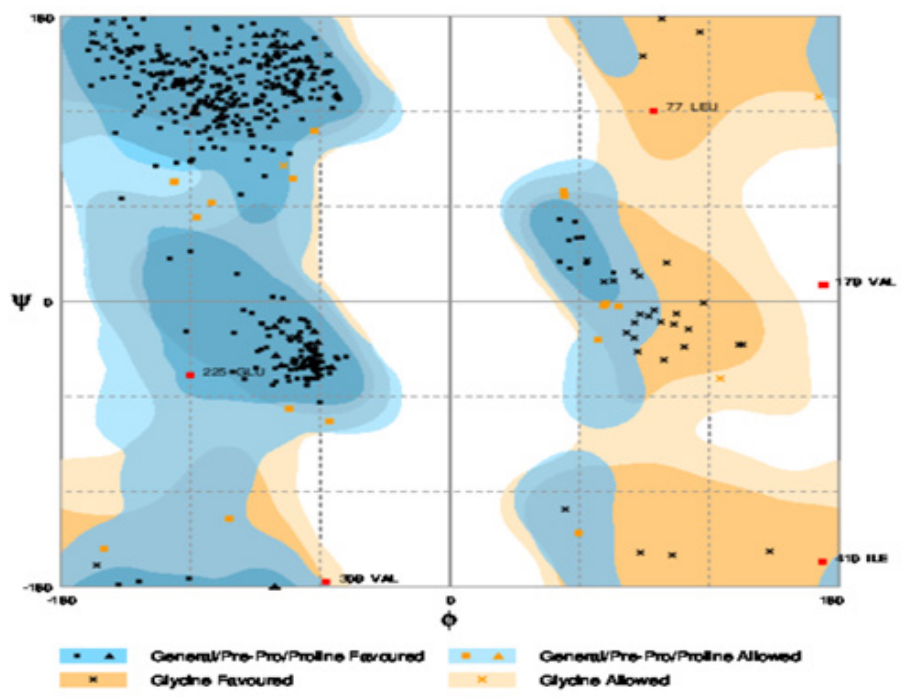

Figure 8: Ramachandran plot of laccase from L. edodes after loop modelling Number of residues in favoured region ( 98.0\% expected):470 (95.1\%) Number of residues in allowed region ( 2.0\% expected):19 (3.8\%) Number of residues in outlier region:5 (1.0\%) 
Table 1: Regions selected for loop modelling.

\begin{tabular}{cc}
\hline Loop number & Amino acid residue \\
\hline 1 & $290-293$ \\
2 & $293-296$ \\
3 & $296-300$ \\
\hline
\end{tabular}

or hazardous organic pollutants released from the pharmaceutical industries. ${ }^{25}$ Mikolasch et al. synthesized the different penicillins through novel coupling process using laccase. ${ }^{26}$ Laccases may be valuable in cancer treatment. In recent years, laccases from different sources have been used in the development of new anticancer medicines and inhibition of proliferation of different types of cells. Authors have discussed prospective roles of laccases in the above mentioned applications. ${ }^{27,28}$ In addition, laccases have been utilized in deactivation study of HIV and hepatitis $\mathrm{C}$ virus that are responsible for acquired immunodeficiency syndrome (AIDS) and hepatitis $\mathrm{C}$ respectively. ${ }^{29,30}$ Hosny et al. utilized the laccase of $R$. vernicifera in the formation of two new catechin- hydroquinone adducts. ${ }^{31}$ These catechins have antioxidant activities and also act as free radical scavenger due to which they have protective roles against cancer, inflammatory and cardiovascular diseases. ${ }^{32,33}$ Lateef et al. synthesized the silver nanoparticles (AgNPs) using crude laccase obtained from an edible mushroom Lentinus edodus. ${ }^{34}$ According to the report, silver nano particles have been utilized in burns treatment, as dental materials, treatment of water, textile fabrics and sunscreen lotions. ${ }^{35}$ In addition, Erb- Downward et al. studied the role of laccase in the production of prostaglandin by C. neoformans. ${ }^{36}$

In this study, the sequence of the laccase from $L$. edodes was retrieved from UniProt KB in FASTA format (Figure 1). BLAST- P server was used to identify the template for laccase from $L$. edodes. The Lac $\mathrm{b}$ from Trametes sp AH28-2 (3KW7A) with 63\% identity was used as template. The secondary structure was predicted by the improved self- optimized prediction method (SOPMA). The results revealed that the proportion of random coils, $\beta$ turns, $\alpha$ helices and extended strands ( $\beta$ folds) accounted for $36.26 \%, 13.17 \%, 18.32 \%$ and $32.25 \%$ of the secondary structure, respectively (Figure 2).

The refined sequence - sequence alignment as obtained by BLAST- P (Figure 3) was used to construct 3D model of laccase from Lentinula edodes using MODWEB server. The model generated from ModWeb was submitted to Verify 3D program and the graph was obtained (Figure 4). Analysis of the graph revealed that some of the regions in the model were not stable and such regions corresponded to the regions of insertion and deletion. These regions were considered for Loop modelling (Table 1). The stereo chemical quality of the model was evaluated using Ramachandran plot (Figure 5). The number of residues in favoured region was 469 (94.9\%), the number of residues in allowed region was 20 (4.0\%) and the number of residues in outlier region was 5 (1.0\%).

Each of the selected loops (Table 1) was remodelled using Swiss PDB Viewer. After loop modelling (Figure 6), verify 3D graph (Figure 7) and stereo chemical quality of the structure by Ramachandran plot (Figure 8 ) were analyzed. The regions of the troughs in the graph (Figure 4) were found to be improved. The number of residues in favoured region is 470 (95.1\%), the number of residues in allowed region is $19(3.8 \%)$ and the number of residues in outlier region is $5(1.0 \%)$. These numbers indicate that the stereo chemical quality of predicted $3 \mathrm{D}$ structure is reasonably good. In addition, the analysis of physical and chemical properties of the laccase from L. edodes was carried out using ProtParam tool.

\section{CONCLUSION}

Laccase from L. edodes has important applications like lignin degradation, pigment biosynthesis, fruiting body formation, detoxification, morphogenesis, as well as pathogenesis. It is also used in hair colouring systems, it has many other agricultural, industrial and medicinal applications. In this study, we have reported the three- dimensional homology model of the laccase from $L$. edodes I, the sequence of which retrieved from Uniprot KB database. The results indicate that the stereo chemical quality and verify $3 \mathrm{D}$ profile of the laccase model is reasonably good. It suggests that this modelled protein can be used to understand the structural and functional properties of laccase from L. edodes. This enzyme has potential for the synthesis of several useful drugs such as anticancerous, antioxidants, synthesis of hormone derivatives because of its high value of oxidation potential. Hence, understanding the threedimensional structure of this enzyme by the above study will enable us to explore the options of above-mentioned drugs. In addition, this modelled protein can also be helpful in exploring the molecular interactions of this laccase with the other proteins.

\section{ACKNOWLEDGEMENT}

The Authors wish to place on record their thanks to the Management of Gokula Education Foundation, Bangalore, India for providing computer laboratory facility and infrastructure for carrying out this research work and the Principal, Head of the Department and the faculty of Department of Microbiology, Ramaiah College of Arts, Science and Commerce, Bangalore, India for their support and cooperation during the course of this work.

\section{CONFLICT OF INTEREST}

The authors declare no conflict of interest.

\section{ABBREVIATIONS}

3D: Three Dimensional; UniprotKB: Uniprot Knowledgebase; BLAST: Basic Local Alignment Search Tool; SOPMA: Self- Optimised Prediction Method with Alignment; PDB: Protein Data Bank; SPDBV: Swiss PDB Viewer; GRAVY: Grand Average of hydropathicity; HIV: Human Immuno Virus; AIDS: Acquired immune deficiency syndrome; AgNps: Silver Nanoparticles.

\section{REFERENCES}

1. Leonowicz A, Cho NS, Luterek J, Wilkolazka A, Wojtas-Wasilewska M, Matuszewska A, et al. Fungal laccase: Properties and activity on lignin. J Basic Microbiol. 2001;41(3-4):185-227.

2. BreeneW. Nutritional and medicinal value of specialty mushrooms. J Food Prot 1990;53(10):883-994.

3. Givaudan A, Effosse A, Faure D, Potier P, Bouillant ML, Bally R. Polyphenol oxidase in Azospirillum lipoferum isolated from rice rhizosphere: Evidence for laccase activity in nonmotile strains of Azospirillum lipoferum. FEMS Microbiol Lett. 1993;108(2):205-10.

4. Ledys SC, Xiomara C, Jorge B, Lopez-Lucendo MF, Maria JM, Susana C Identification and characterization of laccase-type multicopper oxidases involved in dye-decolorization by the fungus Leptosphaerulina sp. BMC Biotechnol. 2015;15-74.

5. Miles PG, Chang ST. Mushrooms: Cultivation, Nutritional Value, Medicinal Effect and Environment Impact. CRC Press. 2004;14:29-45.

6. Faure D, Bouillant ML, Bally R. Isolation of Azospirillum lipoferum 4T Tn5 mutants affected in melanization and laccase activity. Appl Environ Microbiol. 1994;60(9):3413-5.

7. Sydney Brenner, Jeffrey H. Miller, William J. Broughton. Encyclopedia of genetics. Academic Press. 2002; p.1945.

8. Feng Xu, Mary AL. Applications of oxidoreductases: Recent progress. Industrial Biotechnol. 2005;1(1):38-50.

9. DeBakker PIW, DePristo MA, Shetty RP, Blundell TL. Discrete restraint-based modelling and the $\mathrm{C} \alpha$-trace problem. Protein Sci. 2003;12(9):2032-46.

10. Cathy WCHI, Apweiler R, Bairoch A, Natale DA, Barker WC, Boeckmann B, et 
al. The Universal Protein Resource (UniPort)- an expanding universe of protein information. Nucleic Acid Res. 2006;34(Suppl_1):187-91.

11. Basic Local Alignment Search Tool. Available in http://www.ncbi.nlm.nih.gov/ BLAST. Last assessed on 24-09-2020.

12. Altschul SF, Gish W, Miller W, Myers EW, Lipman DJ. Basic local alignment search tool. J Mol Biol. 1990;215(3):403-10.

13. Altschul SF, Madden TL, Schaffer AA, Zhan J, Zhang Z, Miller W, et al. Gapped BLAST and PSI-BLAST: A new generation of protein database search programs. Nucleic Acids Res. 1997;25(17):3389-402.

14. Geourjon C, Deleage G. SOPMA: Significant improvements in protein secondary structure prediction by consensus prediction from multiple alignments. Comput Appl Biosci. 1995;11(6):681-4.

15. Levin JM, Robson B, Garnier J. An algorithm for secondary structure determination in proteins based on sequence similarity. FEBS Lett. 1986:205(2):303-8

16. http://salilab.org/modweb

17. Sali A, Blundell TL. Comparative protein modeling by satisfaction of spatial restraints. J Mol Biol. 1993;234:779.

18. Guex N. Peitsch MC. SWISS-Pdb Viewer: An environment for comparative protein modelling. Electrophoresis. 1997;18(15):2714-23.

19. Roger SE, Milner-White J. Ras Mol: Biomolecular graphics for all. Trends Biochem Sci. 1995;20(9):374.

20. Lüthy R, Bowie JU, Eisenberg D. Assessment of protein models with threedimensional profiles. Nature. 1992;356(6364):83-5.

21. David E, Roland L, James UB. VERIFY3D: Assessment of protein models with three-dimensional profiles. Methods in Enzymology.1997;277:396-404.

22. Ramachandran, G.N., Sasisekharan, V. Conformation of polypeptides and proteins. Adv Protein Chem. 1968;23:283-438.

23. Richardson DC, Lovell SC, Davis IW, Arendall WB, DeBakker PIW, Word JM, et al. Structure validation by Calpha geometry: phi, psi and Cbeta deviation. Proteins: Struct. Funct Genet. 2003;50:437-50.

24. Gasteiger E, Hoogland C, Gattiker A, Duvaud S, Wilkins MR, Appel RD, et al. Protein Identification and Analysis Tools on the ExPASy Server. The Proteomics
Protocols Handbook, Humana Press. 2005;571-607.

25. Pankaj KC, Shashi LB, Chandana S. Laccases in pharmaceutical chemistry: A comprehensive appraisal. Mini Rev Org Chem. 2016;13(6):430-51.

26. Micolasch A, Niedermeyer THJ, Lalk M, Witt S, Seefeldt S, Hammer E, et al. Novel penicillins synthesized by biotransformation using laccase from Trametes spec. Chem Pharm Bull. 2006;54(5):632-8.

27. Eggert C, Temp U, Dean JFD, Erikkson KEL. Laccase mediated formation of the phenoxazinone derivative, cinnabarinic acid. FEBS Lett. 1995;376(3):202-6.

28. Christen S, Thoma SR, Garner B, Stocker R. Inhibition by interferon- gamma of human mononuclear cell-mediated low density lipoprotein oxidation. Participation of tryptophan metabolism along the kynurenine pathway. J Clin Invest. 1994;93(5):2149-58.

29. Wong JH, Ng TB, Jiang Y, Liu F, Sze SCW, Zhang KY. Purification and characterization of a laccase with inhibitory activity towards HIV-1 reverse transcriptase and tumor cells from an edible mushroom (Pleurotuscornucopiae). Protein Pept Lett. 2010;17(8):1040-7.

30. Fakharany ELEM, Haroun BM, Ng TB, Redwan ELRM. Oyster mushroom laccase inhibits hepatitis $C$ virus entry into peripheral blood cells and hepatoma cells. Protein Pept Lett. 2010;17(8):1031-9.

31. Hosny M, Rosazza JPN. Novel oxidations of $(+)$-catechin by Horseradish peroxidase and laccase. J Agric Food Chem. 2002;50(20):5539-45

32. Yang CS, Landau JM. Effects of tea consumption on nutrition and health. J Nutr. 2000;130(10)2409-12.

33. Croft KD. Antioxidant effects of plant phenolic compounds. In: Antioxidants in human health and disease. CABI Publishing: New York. 1999;109-21.

34. Lateef A, Adeeyo AO. Green synthesis and antibacterial activities of silver nanoparticles using extracellular laccase of Lentinusedodes. Not Sci Biol. 2015;7(4):405-11

35. Duran N, Marcato PD, De S, Gabriel IH, Alves OL, Esposito E. Antibacterial effect of silver nanoparticles produced by fungal process on textile fabrics and their effluent treatment. J Biomed Nanotechnol. 2007;3(2):203-8.

36. Downward JR, Huffnagle GB. Cryptococcus neoformans produces authentic prostaglandin E2 without a cyclooxygenase. Eukaryot Cell. 2007;6(2):346-50.

Article History: Submission Date : 26-02-2020; Revised Date : 08-04-2020; Acceptance Date : 26-07-2020.

Cite this article: Vani V, Jayashree DJ, Navya KS, Akanchha, Zaman ST. Homology Modelling and in silico Characterization of Laccase from Lentinula edodes. Int. J. Pharm. Investigation, 2020;10(3):273-8 